\title{
EMANCIPACIÓN E IGUALDAD: ASPECTOS SOCIOPOLÍTICOS DE UNA EXPERIENCIA PEDAGÓGICA
}

\author{
FRANCISCO JÓDAR \\ LuCía GÓMEZ
}

\begin{abstract}
RESUMEN: Una vez detallada la nueva constelación de sentido sobre la educación que produce la experiencia pedagógica de Joseph Jacotot llamada "enseñanza universal" y tras caracterizarla en tanto que acontecimiento, el presente texto aborda algunos aspectos sociopolíticos que ahí están presentes. Dicho abordaje utiliza el utillaje conceptual de autores contemporáneos, especialmente Bourdieu, Deleuze y Foucault. Y pretende desgranar la singularidad de la actuación educativa de Jacotot y de su pensamiento sobre la emancipación y la igualdad. De este modo, se presenta un Jacotot cuya postura sobre la educación, la emancipación y la igualdad, a la par que se distanciaba de las creencias dominantes en su época, sostiene supuestos básicos que guardan especial resonancia con el pensamiento sociopolítico contemporáneo.
\end{abstract}

Palabras clave: Educación. Igualdad. Emancipación. Política.

\section{EMANCIPATION AND EQUALITY: SOCIOPOLITICAL ASPECTS OF A PEDAGOGICAL EXPERIENCE}

ABSTRACT: After detailing the new constellation of educational meanings brought forth by the pedagogical experience of Joseph Jacotot, called "universal teaching" and characterizing it as an "event", this paper tackles some sociopolitical aspects it comprises. Relying upon the conceptual tools provided by such contemporary authors as Bourdieu, Deleuze and Foucault, it intends to unwrap the singu-

\footnotetext{
* Profesor del Departamento de Didáctica y Organización Escolar de la Universidad de Valencia. E-mail: Francisco.Jodar@uv.es

** Profesora del Departamento de Psicobiología y Psicología Social de la Universidad de Valencia. E-mail: Lucia.Gomez-Sanchez@uv.es
} 
larity of the educational proceedings and thoughts of Jacotot about emancipation and equality. It thus introduces a Jacotot whose views on education, emancipation and equality, while opposing the prevailing beliefs of his time, held basic assumptions that prove to be particularly relevant for contemporary sociopolitical thinking.

Key words: Education. Emancipation. Equality. Politics.

\section{Una experiencia pedagógica}

7 oseph Jacotot es el nombre de un "lector de literatura francesa en la universidad de Louvain" y 1818 el año en el que por azar "emprende una aventura intelectual” (Rancière, 1987/2002, p. 17). Jacotot, 1818 señala así una singular experiencia pedagógica: la aventura de quien hace de la creencia en la igualdad de las inteligencias el punto de partida de su concepción de la educación y la política. Los hechos le han mostrado que los alumnos "aprenden solos y sin maestro explicador" (idem, ibid, p. 29). La afirmación de este hecho da lugar a sus lecciones sobre la emancipación intelectual.

La experiencia Jacotot, 1818 rompe con la oposición ciencia e ignorancia sobre la que se edifica la vieja lógica pedagógica: "antes que ser acto pedagógico, la explicación es el mito de la pedagogía, la parábola de un mundo divido en espíritus sabios y espíritus ignorantes, espíritus maduros e inmaduros, capaces e incapaces, inteligentes y tontos" (idem, ibid, p. 23-24). De esta revelación emerge la posición que Jacotot asume. Por un lado, la crítica al orden explicador sobre el que se funda la pedagogía ("hay embrutecimiento allí donde una inteligencia es subordinada a otra inteligencia", idem, ibid., p. 31) y, por otro lado, la apuesta por la emancipación intelectual entendida como "toma de conciencia por todo hombre de su naturaleza de sujeto intelectual" (idem, ibid., p. 60).

La aventura de Jacotot es así una práctica de resistencia frente a una educación que embrutece ("quien enseña sin emancipar embrutece", idem, ibid., p. 37), dando lugar así a la singularidad de un modo de pensar y hacer educación. Esto es, la creación de una nueva sensibilidad pedagógica que llega a contradecir la propia creencia inicial de un Jacotot "concienzudo profesor explicador", según la cual "la gran tarea del maestro es transmitir sus conocimientos a sus alumnos para conducirlos gradualmente a su propia ciencia" (idem, ibid., p. 19). En efecto, el azar hace experimentar a Jacotot un pliegue creativo sobre sí mismo. Creación que es, a la par, exploración del mundo educativo 
mismo. Producción de una nueva constelación de sentido para la educación: la enseñanza universal.

\section{Una constelación de sentido para la educación}

Jacotot produce una constelación de sentido para la educación cuya estrella polar es la emancipación intelectual: afirmación de la inteligencia de cada hombre en tanto copartícipe en un mundo común. De modo que el reconocimiento de la igualdad de las inteligencias es un supuesto de partida cuya verificación "puede" contribuir a la modificación positiva del orden social desigual y a desnaturalizar las instituciones, sus papeles y posiciones.

Así pues, esta es la estrella polar de la constelación: el trabajo educativo encaminado a que cada cual salga del estado de minoría y así "pueda" inscribirse - adquirir visibilidad y ser escuchado - en el espacio común en tanto ser activo dotado de razón e inteligencia. Esto es, adquisición de la disposición activa para "usar su propia inteligencia" (idem, ibid., p. 34) y actuar en el orden social para verificar la igualdad que ahí de hecho continuamente se desmiente. Si bien, conviene tenerlo presente, se trata de una estrella polar que siempre es "virtual", lo que "no significa que sea ilusoria" sino que, más bien, es "potencia de la que conviene verificar los efectos" (Rancière, 2003, p. 40).

En el centro de la constelación se encuentran dos astros gemelos. Por un lado, el hombre, ser dotado de palabra. Por otro, la actualización de las virtudes igualitarias de la inteligencia humana. Desde el primero se atisba el poder de comprender y hacerse entender como medio "de verificación del otro" (idem, 1987/2002, p. 107), donde la razón no es algo que se tiene en poder ni se transmite, cede u otorga, sino construcción en el concurso igualitario de la capacidad intelectual de cada hombre. Del segundo de esos astros se deriva una particular moral. La moral de los discursos, aquella "que preside el acto de hablar y escribir, aquella de la intención de comunicar, del reconocimiento del otro como sujeto intelectual capaz de comprender lo que otro sujeto intelectual quiere decirle" (idem, ibid., p. 186-187).

Estos dos astros iluminan el espacio donde se inscribe "la confianza en la capacidad intelectual de todo ser humano" (idem, ibid., p. 32). Confianza en que cualquier individuo es capaz, mediante la aventura intelectual correspondiente, de adivinar el sentido de lo que está dicho y escrito. Un sentido cuya verdad no está asegurada ni contenida en ningún código o diccionario, en ninguna explicación. Además la confianza en la igualdad es 
un supuesto de partida al que se ha de responder continuamente: "nuestro problema no es probar que todas las inteligencias son iguales. Sino ver qué se puede hacer con esta suposición" (idem, ibid., p. 72).

A cierta distancia de la constelación se encuentra el orden de las cosas, la sociedad desigual, el círculo social. Esa distancia designa un espacio donde coinciden sin anudarse ni anularse dos lógicas diferentes: la lógica igualitaria implicada en el acto de la palabra y la lógica desigual inherente a la relación social. Un espacio también para experimentar la contingencia de las jerarquías del orden social. Se comprende así, por obra de esta distancia entre órdenes distintos, que "la enseñanza universal no es ni puede ser nunca un método social” (idem, ibid., p. 146). Dicho de otro modo: "no hay principio de la comunidad de iguales que sea principio de organización social" (Rancière, 2003, p. 65-66). En efecto, de la constelación de la emancipación intelectual al orden social no hay tránsito posible sin pasar por una distancia irreductible e insalvable, aquella donde la experiencia individual y colectiva lucha por verificar en el orden de las cosas la igualdad de partida que se presupone en el orden de las lenguas. En este aprender a cruzar esa distancia - ser individuo razonable en un orden social carente de razón - se juega gran parte del sentido que Jacotot ha producido para la educación en la llamada enseñanza universal.

La necesidad de confirmar esa constelación que él una vez experimentó dota de singular fuerza social y política a las enseñanzas que ofrece Jacotot. ¿En qué consiste, entonces, la singularidad de la constelación?

\section{El acontecimiento Jacotot, 1818}

\section{En El maestro ignorante Rancière presenta un Jacotot "loco":}

la singularidad, la locura de Joseph Jacotot, fue sentir esto: era el momento donde la joven causa de la emancipación, la de la igualdad de los hombres, estaba a punto de transformarse en causa de progreso social. Y el progreso social, era en primer lugar el progreso en la capacidad del orden social a ser reconocido como orden racional. Esta creencia no podía desarrollarse más que en detrimento del esfuerzo emancipador de los individuos razonables, al precio del ahogo de las virtualidades humanas que comporta la idea de la igualdad [...] Jacotot fue el único en pensar esta desaparición de la igualdad bajo el progreso, de la emancipación bajo la instrucción. (Rancière, 1987/2002, p. 184)

Si ésta - la condición de "loco" que respecto a la idea de igualdad y emancipación abre el campo de lo dado socialmente por posible - es la pista a seguir, la pregunta que no podemos obviar es la siguiente: 
¿qué carácter tiene la experiencia pedagógica de Jacotot?, ¿por qué su singularidad deshace el mundo de la educación de su época?, ¿por qué supone un enfrentamiento sin paliativos a los problemas de la emancipación? Diremos que ello es así porque la experiencia pedagógica de Jacotot supone la efectuación de un acontecimiento.

Siguiendo a Derrida (1992, p. 39), la experiencia-experimentación Jacotot, 1818 tiene que ver con hacer "experiencia de lo imposible" en educación; o en términos de Deleuze (1987, p. 128), con la confrontación con algo "impensado" en el pensamiento pedagógico. Por eso, la experiencia Jacotot, en tanto acontecimiento, está escrita "bajo la atracción de lo real imposible" (Blanchot, 1987, p. 39). Es una experiencia que exige ir donde es imposible ir (enseñar lo que no se sabe) y pensar lo que no se deja pensar (la creencia en la igualdad como necesidad de una enseñanza universal no jerarquizadora que es negada por la instrucción pública). Lo que, por otro lado, también es un modo de nombrar la condición intempestiva del acontecimiento Jacotot, 1818. Según la concepción genealógica de la historia de Foucault (1988), el acontecimiento es experiencia de una heterogeneidad radical, de modo que su conocimiento, al introducir las discontinuidades en lo que somos, no dice lo que somos sino aquello de lo que diferimos, no establece nuestra identidad sino aquello que la disipa.

Así pues, en tanto acontecimiento, la experiencia de Jacotot no supone en modo alguno la realización de un método pedagógico posible entre otros: "se trata de osar a aventurarse y no de aprender más o menos bien ni más o menos deprisa. El 'método Jacotot' no es el mejor, es otro" (Rancière, $1987 / 2002$, p. 49). No se ofrece como una opción ni se escoge; su efectuación no deja un resto de alternativas descartadas o por cumplir. La efectividad del acontecimiento Jacotot, 1818 es un modo de pensar y hacer educación que resulta de una alteridad radical a los parámetros reconocibles de la educación instituida y su "viejo método". En este sentido, su efectividad no se define por lo que realiza sino por lo que abre y da lugar.

\section{Las afueras de la instrucción y del progreso}

¿Qué abre Jacotot en su aventura? ¿Qué impensado del pensamiento educativo acoge su exploración? Destacamos dos elementos entrelazados.

Jacotot, en primer lugar, establece un espacio de conflicto donde se separan y encuentran dos procesos heterogéneos. Por un lado, el juego de prácticas educativas guiadas por la presuposición de la igualdad de cualquiera y por la preocupación de verificar tal presupuesto. Por otro, las instituciones educativas que producen consentimiento a una distribución jerárquica de cuerpos, lugares y funciones. 
El nombre que Jacotot asigna al primero de los procesos es el de emancipación. Al segundo de ellos pertenece la instrucción pública. De este modo la emancipación queda circunscrita a la esfera de los individuos: "la enseñanza universal no puede dirigirse más que a individuos, jamás a sociedades" (idem, ibid., p. 146), pues "jamás ningún partido gobernante, ningún ejército, ninguna escuela ni ninguna institución emancipará a una sola persona" (idem, ibid., p. 142). Y la instrucción participa en la propagación y consolidación de la sinrazón de las desigualdades: "una sociedad, un pueblo, un Estado, serán siempre desrazonables" (idem, ibid., p. 140).

Acaso para nombrar ambos procesos puedan ser de utilidad dos conceptos de Foucault: discusión y polémica. Dos conceptos que suponen dos morales distintas respecto "a la búsqueda de la verdad y a la relación del otro". Así, el proceso de igualdad-emancipación acoge la discusión, esto es, el "trabajo de elucidación recíproca" (Foucault, 1999, p. 353), donde los derechos de quien pregunta y de quien responde "son de algún modo inmanentes a la discusión" misma. Ningún interlocutor es poseedor de derecho alguno que exceda al desarrollo de la discusión. Por otro lado, la instrucción pública y la explicación es una modalidad de la polémica. En ella uno se "aproxima acorazado de privilegios que ostenta de entrada y que nunca acepta poner en cuestión", para lo cual "se apoya en una legitimidad de la que, por definición, es excluido su adversario" (idem, ibid, p. 353354). Aquí el interlocutor no es alguien con quien buscar la verdad ni sujeto reconocido con derecho a la palabra, es adversario, enemigo cuya existencia misma constituye una amenaza. Son tres las modalidades de polémica que Foucault reconoce: la religión, con sus dogmas que el adversario no puede ni ignorar ni transgredir; la judicial, con la instrucción de un proceso donde el interlocutor es un sospechoso y en la que hay que reunir pruebas para su condena o absolución; la política, con sus intereses de partidos, donde el otro es un enemigo a vencer. A estos tres modelos de polémica, siguiendo a Jacotot, cabe añadir el pedagógico, con sus efectos de embrutecimiento y su explicación jerarquizadora: la división de las inteligencias en inferiores y superiores; la de los niños y hombres del pueblo, por un lado, y, por otro, la del profesor, "conocedor de las cosas por las razones", transmisor "de sus conocimientos adaptándolos a las capacidades intelectuales del alumno" y comprobador "de que el alumno ha comprendido bien lo que ha aprendido" (Rancière, 1987/2002, p. 24).

Jacotot señala, en segundo lugar, algunas de las contradicciones y aporías del intento de invocar "el pueblo" y ponerlo en el centro de la instrucción pública. Así la emancipación intelectual que nos propone se deja leer como la tentativa de quien es consciente que "los pueblos no 
preexisten" (Deleuze, 1995, p. 201), y que, por lo tanto, en educación del pueblo lo único que cabe es experimentar las insospechadas "virtualidades" que se producen una vez que se hace de la comunidad de iguales una comunidad sin dogmatismos ni esencialismos. Comunidad irrepresentable e incontable, sólo sustentada por un presupuesto: la igualdad de la inteligencia de todo hombre, de cualquiera.

Cuando los pueblos no preexisten la tarea es "inventar un pueblo que falta” (Deleuze, 1996, p. 15). El pueblo, por tanto, se hace; no preexiste, se crea. Y la educación no puede consistir en enseñar lo que el pueblo es. Tampoco en transmitirle su verdad ni iluminarlo. La educación no es servidora de pueblo alguno. La educación - cuando es emancipación - tiene el efecto de reconstruir y redefinir sin fin el pueblo siempre ausente y por venir. La educación de los hombres cualesquiera que hacen uso de la razón verificando su igualdad. Lugar de lo universal, donde la demostración - necesariamente provisional - de la igualdad se verifica.

\section{Emancipación y práctica de la igualdad}

Jacotot, tal y como venimos señalando, se enfrenta al modo en el que sus contemporáneos conciben la igualdad y la emancipación. Cuestiona la traducción que se ha hecho de igualdad por progreso y de emancipación por instrucción del pueblo. Muestra que la misma idea de instrucción pública reposa sobre el principio de la desigualdad de las inteligencias. La instrucción del pueblo empírico programada por los representantes del concepto soberano de pueblo - es decir, la dirección de los ignorantes por aquellos que saben, de individuos encerrados en su particularismo por el universal de la razón, de una multitud estúpida por una casta inteligente - presupone dar a ese pueblo al que apelan y en nombre del cual dicen actuar la medida de su propia incapacidad. De este modo, se inicia un proceso de pedagogización integral de la sociedad, de "infantilización general de los individuos que la componen" (Rancière, 1987/2002, p. 183) que tiene como fin reducir progresivamente la desigualdad. Proceso que toma como punto de partida la ignorancia, la incapacidad del pueblo y que tiene como fin promover la igualdad, una igualdad abstracta, "representada, socializada, desigualizada, buena para ser perfeccionada, es decir, retrasada de comisión en comisión, de relación en relación, de reforma en reforma hasta la consumación de los tiempos" (idem, ibid., p. 184).

Frente a esta concepción, Jacotot dibuja un territorio que no renuncia a pensar la posibilidad de igualdad y emancipación lejos del binomio progreso-instrucción. A pesar de que el propio Rancière ha dejado patente sus diferencias con Bourdieu - especialmente con el "nivel de generalidad" 
que sus tesis sociológicas sobre la educación "ha ganado fama entre la doxa política" (Ranciére, 2003, p. 42) - en este punto consideramos que es posible presentar ese territorio sobre la igualdad y la emancipación dibujado por Rancière-Jacotot en conexión con planteamientos de Bourdieu. Conexión que se sostiene en las críticas que ambos han realizado a algunos lugares comunes a los que se recurre para pensar la política.

Bourdieu (1999) abandona la tradición intelectualista de las filosofías de la conciencia con su énfasis en las representaciones, mostrando que las relaciones de dominación se somatizan, es decir, se inscriben y atraviesan la subjetividad; constituyendo así los esquemas mismos de percepción, valoración y acción. La aceptación de las categorías del orden simbólico (que son los principios de visión y división dominantes) supone para determinadas posiciones incorporar una visión desvalorizada de sí mismas, incorporar un "coeficiente simbólico negativo" que afecta de manera negativa a lo que son y a lo que hacen. A esta desigualdad básica que preside las relaciones entre distintas posiciones se refiere Bourdieu con el concepto de violencia simbólica; una violencia invisible pero que adquiere a menudo la forma de la emoción corporal (vergüenza, timidez, impotencia, culpabilidad) y se revela en manifestaciones visibles (sonrojo, turbación verbal, torpeza, temblor...), expresiones del sometimiento al juicio dominante, más allá de las directrices de la conciencia y la voluntad. Se trata de síntomas o manifestaciones que Jacotot coloca también en el centro de su diagnóstico político. Así la propuesta de Jacotot se centra no en la ignorancia sino en la impotencia, "el desprecio de sí, en el desprecio en sí de la criatura razonable" (Rancière, 1987/2002, p. 142) o "la posición humillada" (idem, ibid., p. 147) que caracteriza a aquellos sobre los que pesa masivamente el prejuicio de la desigualdad de la inteligencia.

Bourdieu (1985) considera que la política comienza con la denuncia del contrato tácito de adhesión al orden establecido. Si el orden simbólico no es neutro sino que favorece a unos grupos sobre otros, transforma la arbitrariedad cultural en natural, legitima unas posiciones y deslegitima otras, ratificando, en definitiva, una determinada lógica de dominación, entonces la subversión política presupone una subversión cognitiva, una reconversión de la visión del mundo. Así, las posiciones dominadas sólo pueden movilizarse y movilizar la fuerza que detentan a condición de poner en tela de juicio las categorías de percepción y valoración del orden social que les relegan a una posición subordinada. Por ello, las luchas políticas son luchas simbólicas (Bourdieu, 1999). Para Jacotot la emancipación, esto es, "que todo hombre del pueblo pueda concebir su dignidad de hombre, tomar la medida de su capacidad intelectual y decidir su uso" (Rancière, 1987/2002, p. 37) exige, del mismo modo, cuestionar lo que denomina "la visión embrutecedora del 
mundo" que sostiene la realidad de la desigualdad y, frente a ella, comprender que el orden social, sus clasificaciones y rangos, es sólo "convención", "ficción" (idem, ibid., p. 180).

Bourdieu, al problematizar la relación entre poder y subjetividad, señala los límites y las contradicciones de los esquemas modernos (representación, progreso, igualdad abstracta...) que han guiado la lucha y el pensamiento político. Frente a la idea de progreso en la que opera la escisión entre medios y fines, desde esta posición, la política, el espacio de la luchas simbólicas, es práctica política, fin en sí misma porque supone inaugurar discursos y relaciones que desmientan las divisiones jerárquicas que recorren el orden social. La igualdad, aquí, deja de formar parte de un horizonte lejano y se convierte en algo que debe ser practicado. Del mismo modo, Jacotot propone, a través de la acción sobre el juego estratégico de relaciones existente "hacer circular la energía eléctrica de la emancipación" (idem, ibid., p. 180) en el cuerpo social, de modo que se desborde el presupuesto que sostiene el proceso de instrucción: la desigualdad de las inteligencias. Prácticas y relaciones que no son un medio para obtener la igualdad, para un fin situado en otro lugar, sino que tienen efectos reales en sí mismas, constituyen la "verificación" de la igualdad.

Admitir esos puntos de conexión, a menudo desapercibidos, no imposibilita apreciar que el trabajo intelectual de Bourdieu y RancièreJacotot toman derivas distintas. Si el reto de Rancière (lector-escritor, por ello, de Jacotot) es verificar la potencia de la igualdad, Bourdieu dirige sus esfuerzos a mostrar las dificultades de la política, a analizar sus condiciones de (im)posibilidad. De ahí el papel que Bourdieu otorga a las ciencias sociales: analizar racionalmente la dominación. Bourdieu (2000) quiere hacer visible que, dependiendo de las posiciones en el espacio social, los agentes involucrados en la lucha se encuentran muy desigualmente armados en la batalla por imponer su verdad; es decir, la propia labor simbólica necesaria para entrar en la lucha política (enunciar la arbitrariedad del orden social) supone unos instrumentos de expresión y crítica que están desigualmente distribuidos. Y no sólo eso, para Bourdieu (1999) la lucha política debe cuestionar tanto el orden de los cuerpos (disposiciones) como el orden de las cosas (instituciones). Dicho de otro modo: se trata de dar fuerza social a la crítica intelectual y fuerza intelectual a la crítica social.

Frente a estos postulados, Rancière cuestiona la crítica social llevada a cabo por Bourdieu entendida como dispositivo de análisis que desmitifica la creencia en la igualdad. En este sentido Rancière ha señalado los efectos perversos de este "pensamiento de la sospecha" en el plano político en la medida en que parten de la verificación de la desigualdad. Por eso Rancière se distancia de la apropiación "progresista” que pedagogos y políticos han realizado de la tesis de Bourdieu en sus intentos por hacer de la escuela un 
instrumento institucional de reducción de desigualdades. Para Rancière esta interpretación política derivada del pensamiento de la sospecha ha tenido un claro resultado: "pretendiendo explicar la desigualdad se la ha vuelto más rígida” (Ranciére, 2003, p. 43).

No obstante, es interesante resaltar que ambos nos proponen que una politica de la experiencia o una práctica de la igualdad - que implica ponerse en juego, experimentarse, ensayarse, probarse - sustituya a la utopía. De ahí que imaginar y practicar los efectos subjetivos, sociales y políticos desencadenados en los contextos más variados que originaría la "subversión cognitiva" que propone Bourdieu o la posibilidad de "despertar en el hombre social el hombre razonable" (idem, 1987/2002, p. 150) que sostiene Jacotot sigue siendo hoy un desafío al que responder.

Recebido e aprovado em fevereiro de 2003.

Referencias bibliográficas

BLANCHOT, M. La escritura del desastre. Caracas: Monte Ávila, 1987. BOURDIEU, P. ¿Qué significa hablar? Madrid: Akal, 1985.

BOURDIEU, P. Poder, derecho y clases sociales. Bilbao: Desclée de Bouwer, 2000.

BOURDIEU, P. Meditaciones pascalianas. Barcelona: Anagrama, 1999.

DELEUZE, G. Foucault. Barcelona: Paidós, 1987.

DELEUZE, G. Conversaciones. Valencia: Pre-Textos, 1995.

DELEUZE, G. Crítica y clínica. Barcelona: Anagrama, 1996.

DERRIDA, J. El otro cabo/La democracia, para otro día. Barcelona: Ed. del Serbal, 1992.

FOUCAULT, M. Nietzsche, la genealogía, la historia. Valencia: Pre-Textos, 1988.

FOUCAULT, M. Polémica, política y problematizaciones. In: FOUCAULT, M. Estética, ética y hermenéutica. Barcelona: Paidós, 1999, p. 353-361.

RANCIÈRE, J. Le maître ignorant. Cinq leçons sur l'émancipation intellectuelle. Paris: Fayard, 1987. Las citas corresponden a la edición en portugués (Belo Horizonte: Autêntica, 2002).

RANCIÈRE, J. En los bordes de lo político. Disponible en <http:// www.philosophia.cl/Biblioteca/Rancieres. Traducción de Alejandro Madrid-Zan y José Grossi. Acceso en 20 enero. 2003. 